\title{
Fluid dynamics of evolving foams ${ }^{\dagger}$
}

\author{
Raquel Verdejo, ${ }^{* a}$ Francisco J. Tapiador, ${ }^{b}$ Lukas Helfen, ${ }^{c}$ M. Mar Bernal, ${ }^{a}$ Natacha Bitinis ${ }^{\mathrm{a}}$ and Miguel \\ A. Lopez-Manchado ${ }^{a}$
}

5

The physical properties of many multiphase systems are determined by coarsening phenomena. From raindrops to polycrystal grains and foams, the formation and stability of these systems continuously evolve towards lower-energy configurations through events such as coalescence, Ostwald ripening and drainage. Here we propose a procedure to identify and characterise key 10 topological transformations of coarsening phenomena using a physically-based fluid dynamic framework. In-situ, real-time foaming processes of a polymeric matrix reinforced with two morphologically different nanofillers, carbon nanotubes and graphene sheets were observed by synchrotron $\mathrm{x}$-ray radioscopy. We obtained detailed information on the evolution of the growth patterns and coarsening events. Filled samples showed differences in both trend and speed

15 compared with the unfilled sample. Furthermore, we found different dominating coarsening phenomena due to the wetting nature of carbon nanoparticles. Our procedure can be extended to sequences of any type of $2 \mathrm{D}$ projection or $3 \mathrm{D}$ images and to other multiphase systems.

\section{Introduction}

Foaming is a complex process which involves the formation 20 of gas bubbles in a fluid or nucleation and the evolution of both liquid and gas until stabilization occurs. This spatial and temporal evolution involves the interplay of several physical phenomena such as surface tension, diffusion and viscosity ${ }^{1}$ which is reflected in the dynamics of the liquid and gaseous 25 phases and determines the final cellular microstructure of the system.

Despite the important influence that this cellular topology has on the properties of foams ${ }^{2}$, the physics of the foaming phenomena, such as drainage and foam flow, are still unclear, 30 even in the simplest case of the aqueous foams. ${ }^{3}$ The majority of current studies focusing on foam formation have been carried out on aqueous foams by light scattering techniques ${ }^{4-6}$ or AC conductivity. ${ }^{7}$ Ideally, the same basic theories should apply to aqueous and non-aqueous foams. ${ }^{8}$ However, recent 35 studies of the foaming of metal foams involving synchrotron analyses have shown clear differences between aqueous and non-aqueous foams. ${ }^{9}$ Banhart et al. ${ }^{9}$ showed that drainage due to gravity had only a weak effect on coarsening phenomena affecting metal foams while interdependence was observed 40 between drainage and coarsening in aqueous foams. ${ }^{3,10,11}$

Here, we report a procedure based on a fluid dynamic framework to identify and characterize key topological transformations in reactive polymer foams. We studied a polymer foam produced by a reactive foaming process. ${ }^{12}$ This 45 process involves the simultaneous foaming and polymerization of liquid reactants, presenting a liquid-solid phase transition (see Supplementary Video, S1). The foaming is driven by a gas generated as a by-product of the polymerization reaction. This reaction has to be well 50 controlled in order to balance the rates of both the evolution of the gas and the increase in viscosity $;^{13}$ if not, the foam will collapse. One practical way in which to improve the stability of reactive foams is to increase the bulk viscosity of the reactants or the surface viscosity of the gas-liquid interface ${ }_{55}$ through a colloidal suspension of solid particles. ${ }^{8}$ These dispersed particles can also act as nucleation sites for bubbles in the early stages of the process and as functional fillers for the final foam.

We have recently studied the use of nanofillers as 60 reinforcements, as they can be physically introduced into cell walls without disrupting the foam cellular microstructure. ${ }^{14,15}$ Electron microscopy of the foams confirmed that the nanofillers were completely embedded and homogeneously dispersed within the polymer matrix, but also showed a drastic 65 change in the cellular microstructure: from isotropic interconnected open pores to highly anisotropic closed pores.

To better understand the foaming evolution and its dynamics, we carried out in-situ foaming experiments using synchrotron radiation; because conventional x-ray sources do 70 not provide the appropriate spatial and temporal resolutions. Synchrotron radiography (SR) has previously been used to study a broad range of physical and technological phenomena: from fuel injection in a running engine, ${ }^{16}$ to formation of granular jets ${ }^{17}$ and foaming of metal systems. ${ }^{9}$ For foaming 75 studies and appropriate spatial sampling distances in the order of several $\mu \mathrm{m}$, SR allows temporal sampling to be adjusted over a wide range from fractions of seconds, using monochromatic radiation, ${ }^{9}$ down to sub-milliseconds, using white radiation. ${ }^{18}$ Depending on the sampling conditions 80 chosen, ${ }^{19}$ X-ray radiography permits the observation and discrimination of events in real time using a series of projected radiographs.

Taking into consideration the significance of the morphology and wetting nature of the particles on the foam 85 stabilization, ${ }^{8}$ we selected two different carbon based nanofillers: carbon nanotubes (CNTs) and functionalised graphene sheets (FGS). As-produced CNTs are non-wetting 
particles, while FGS contain functional groups, epoxy and hydroxyl, ${ }^{20}$ that will enable direct covalent bonding between the filler and the matrix.

\section{Experimental}

\section{${ }_{5}$ Materials and Methods}

Functionalised graphene sheets were produced from the adiabatic expansion of graphite oxide synthesised from natural graphite by the Brodie method. Vapour-grown CNTs were supplied by Bayer, Germany (Baytubes ${ }^{\circledR}$ C $150 \mathrm{P}$ ). The as10 received MWCNTs had mean diameters of $13.5 \pm 6.4 \mathrm{~nm}$ and were free of carbonaceous contamination. The CNTs were used as received, without any further treatment. The polymer matrix was polydimethylsiloxane (PDMS) foam from Bluestar Silicones (Rhodorsil RTFoam 3240) without any commercial 15 filler. The system was composed of two reactants: a silane $(\equiv \mathrm{SiH})$ and a silanol ( $\equiv \mathrm{SiOH}-)$ compound, which contained the $\mathrm{Pt}$ catalyst. PDMS foams were obtained from the condensation reaction between the $\mathrm{SiOH}$ on hydroxylterminated polydimethylsiloxane and the $\mathrm{SiH}$ on 20 polymethylhydrogensilane in the presence of the catalyst and with the evolution of hydrogen. Crosslinking took place as a result of the addition-cure reaction of vinyl endblocked groups with the $\mathrm{Si}-\mathrm{H}$ groups.

The nanofillers were dispersed in ethanol $(10 \mathrm{ml})$ for $5 \mathrm{~min}$, 25 using an ultrasonication probe in an ice bath. This mixture was subsequently dispersed in the $\equiv \mathrm{SiH}$ compound under high-shear mixing to prevent inhibition of the Pt catalyst present in the other compound. At the same time, the ethanol was evaporated until a constant weight was achieved. Finally, 30 the $\equiv \mathrm{SiOH}$ compound was manually mixed with the nanofiller/ $\equiv \mathrm{SiH}$ compound at a $1: 1$ ratio for $1 \mathrm{~min}$ while the safety protocol at ESRF was followed. Foams were produced containing 0 and 0.2 wt.- $\%$ CNT and FGS. The foaming reaction takes place at room temperature as an exothermic 35 reaction. Previous studies ${ }^{14,} 15$ had shown the quality of nanofiller dispersion and the enhanced properties of the nanocomposite foams.

High-intensity synchrotron $\mathrm{x}$-ray radioscopy was used to obtain real-time images of the foaming. The radiograph 40 sequences were produced on beamline ID19 at the European Synchrotron Radiation Facility (ESRF, Grenoble, France). A monochromatic synchrotron X-ray beam $(20 \mathrm{keV})$ was used to acquire the radiographic image sequences (pixel size $2.8 \mu \mathrm{m}$ ), with frame rates of $11 \mathrm{~s}^{-1}$. Sequence acquisition for all 45 samples began 2 minutes after mixing.

\section{Optical flow}

The algorithm proposed to characterize foam evolution was a multi-scale version of the Horn and Schunk optical flow algorithm. $^{21}$ The rationale behind using an optical flow 50 algorithm was that under suitable simplifications, the process could be expressed in terms of the formalism of fluid dynamics, locally satisfying Navier-Stokes equations. ${ }^{22}$ Simpler procedures such as correlation-windows approaches do not allow for sub-pixel estimates of the velocity. The 55 method also permitted sub-pixel accuracy in the estimates, which is a must for the fast frame rates provided by synchrotron imaging.

In order to make sure that the calculations remained related to physical quantities, image operations in the 2D radiographs 60 were used to infer the actual flow (projected onto the image plane) as follows: The velocity vector $\vec{v}$ from point $P_{1}=\vec{x}=\left(x_{1}, x_{2}\right)$ to point $P_{2}=\vec{y}=\left(y_{1}, y_{2}\right)$ (Cartesian coordinates frame) in the radiographs is given by

$$
\vec{v}=(\vec{y}-\vec{x}) / t
$$

${ }_{65}$ Ignoring rotation and shearing in the direction of the beam, in $2 \mathrm{D}$ the motion of a material feature from $\mathrm{P}_{1}$ to $\mathrm{P}_{2}$ in the unit time $(t=1)$ was seen as an affine operation composed of a translation and a linear transformation (rotation, scaling or shear). Using matrix algebra, it was possible to describe affine 70 operations in the image plane as $\vec{y}=A \vec{x}+B$, where $\mathrm{A}$ is a first-order linear transform matrix and $\mathrm{B}$ a first-order translation matrix. Hence, the velocity vector yields

$$
\vec{v}=A \vec{x}+B-\vec{x}=(A-\mathbf{1}) \vec{x}+B
$$

where $\mathbf{1}$ is the identity matrix. Quantitatively, this can be seen 75 as a pointwise image transformation between two successive images. Deriving (2) results in:

$$
\begin{aligned}
& \frac{D \vec{v}}{D t}=\frac{D[(A-\mathbf{1}) \vec{x}+B]}{D t}= \\
& \frac{D[(A-\mathbf{1}) \vec{x}]}{D t}+\frac{D B}{D t}= \\
& \frac{D[(A-\mathbf{1})] \vec{x}+(A-\mathbf{1}) D \vec{x}}{D t}+\frac{D B}{D t}
\end{aligned}
$$

As A and B are first-order matrices, their derivatives are zero:

$$
\frac{D B}{D t}=0
$$

80

$$
D(A-\mathbf{1})=0
$$

So (3) becomes:

$$
\frac{D \vec{v}}{D t}=\frac{(A-\mathbf{1}) D \vec{x}}{D t}=(A-\mathbf{1}) \vec{v}
$$

Applying now the Laplacian, we found that:

$$
\nabla^{2} \cdot \vec{v}=\nabla^{2} \cdot[(A-\mathbf{1}) \vec{x}+B]
$$

${ }_{85}$ In terms of image treatment, the Laplacian is equivalent to the convolution operator using a moving window. The divergence of the gradient for a first-order affine matrix is zero. Thus,

$$
\nabla^{2} \cdot \vec{v}=0
$$

We can now link the physical form of the Navier-Stokes 90 equations with the mathematical treatment of the images.

Navier-Stokes equations read: 


$$
\rho \frac{D \vec{v}}{D t}=-\nabla \cdot p+\mu_{f} \nabla^{2} \cdot \vec{v}+\rho \vec{g}
$$

where $\mu_{\mathrm{f}}$ is the fluid's viscosity. Deriving the velocity vector with respect to time, applying (5) and (7), and considering that the $g$ vector is comparatively small to the other term, results in:

$$
5
$$

$$
\begin{gathered}
\rho(A-\mathbf{1}) \vec{v}=-\nabla \cdot p+\rho \vec{g} \\
\nabla \cdot p=-\rho(A-\mathbf{1}) \vec{v}
\end{gathered}
$$

Equation 9 relates a physical quantity of the system and the pressure gradient with a linear combination of the velocity components seen in the images. A calculation of $\vec{v}$ can now 10 be made based on beam intensity I. As the intensity of the monochromatic beam absorbed/scattered by a material element remains constant between two successive frames:

$$
I\left(x_{1}, x_{2}, t\right)=I\left(x_{1}+\delta x_{1}, x_{2}+\delta x_{2}, t+\delta t\right)
$$

Deriving and referring to the terms as

$$
\begin{aligned}
& d x_{1} / d t=u, d x_{2} / d t=v, \\
& \partial I / \partial x_{1}=I_{x 1}, \partial I / \partial x_{2}=I_{x 2}, \quad \partial I / \partial t=I_{t}, \\
& \text { results in } I_{x 1}+I_{x 2}+I_{t}=0 .
\end{aligned}
$$

Components $\mathrm{u}$ and $\mathrm{v}$ cannot be locally determined as there are two unknowns for each point in time $\left(\mathrm{x}_{1}, \mathrm{x}_{2}, \mathrm{t}\right)$. However, if 20 we assume the local spatial or spatio-temporal constancy of the velocity field, we can define an additional constraint. Thus, we define a function $J$ as the total error committed plus the departure from smoothness in the velocity flow:

$$
J=\sum \sum\left[\alpha\left(I_{x 1} u+I_{x 2} v+I_{t}\right)+\left(\frac{\partial^{2} u}{\partial x_{1}{ }^{2}}+\frac{\partial^{2} u}{\partial x_{2}{ }^{2}}+\frac{\partial^{2} v}{\partial x_{2}{ }^{2}}+\frac{\partial^{2} v}{\partial x_{2}{ }^{2}}\right)\right]=0
$$

25 where $\alpha$ is a weighting factor that depends on the signal-tonoise ratio of the images and on the quantization error of the synchrotron beam that results from data being recorded with finite precision, as determined by the dynamical range of the detector. Here we used an empirically-determined value of ${ }_{30} \alpha=0.1$. The dependence of the results with $\alpha$ is in the form of increased/decreased spatial resolution with lower/larger values of $\alpha$, but the resulting variability affects minimally to the results reported here.

The last step of the method is to use the Lagrange 35 multiplier method to minimize $J$. The result is:

$$
\begin{aligned}
& \frac{\partial u}{\partial t}=\nabla^{2} \cdot u-\alpha I_{x 1}\left(I_{x 1} u+I_{x 2} v+I_{t}\right) \\
& \frac{\partial v}{\partial t}=\nabla^{2} \cdot v-\alpha I_{x 2}\left(I_{x 1} u+I_{x 2} v+I_{t}\right)
\end{aligned}
$$

This pair of equations can now be used at each point of the image to find the components $\mathrm{u}$ and $\mathrm{v}$ of the velocity field. Equation 12 is iteratively solved using the Gauss-Seidel 40 algorithm, resulting in a dense velocity field. The algorithm is applied to a Gaussian pyramid decomposition of the images in order to provide first-guess values to the iteration at lower scales, which makes it possible to capture large scale displacements. The method can be described as a linear 45 expansion of the Navier-Stokes equations.

\section{Procedure}

The foaming process was typically recorded in one thousand high-resolution radiographs (see Supplementary Video, S2). ${ }_{50}$ Qualitative exploration of the images ensured the suitability of the dataset for further exploration. Quantitative analysis was carried out using a physically-based optical flow algorithm. The analysis procedure is illustrated in Figure 1.

First, the raw x-ray images were normalised using beam 55 reference and dark images to filter out both beam and detector artefacts. From these radiographs, $\mathrm{r}(\mathrm{x}, \mathrm{y})$, we were able to calculate the line integrals in the direction of the beam for each pixel according to the formula: $I(x, y)=-\ln (r(x, y)) / \mu_{x}(E)$, because we worked with quasi-monochromatic radiation of ${ }_{60}$ energy E. $\mu_{\mathrm{x}}(\mathrm{E})$ refers to the linear absorption coefficient of the monochromatic beam, conventionally defined as the inverse of the mean path after which the beam has been attenuated to $1 / \exp (1)(\approx 37 \%)$ of its initial intensity. This formula provides a mean to linearly relate the intensity of the 65 signal to the material density and solves the beam hardening effect due to the exponential decrease of the intensity of $\mathrm{x}$ rays as they pass through a material. ${ }^{23}$ For a dispersion in a fluid (which has been shown homogeneous on the scale of the imaging resolution, ${ }^{14,} 15$ i.e. in our case on the $\mu \mathrm{m}$ scale), and 70 a non-absorbing gas $\left(\mu_{x}=0\right)$, the quantity $\mathrm{I}(\mathrm{x}, \mathrm{y})$ can be interpreted as the thickness of the fluid along the $\mathrm{x}$-ray beam direction. For each of the resulting projection images $I(x, y)$, we then calculated the difference between each image and its successor in order to highlight the structural changes inherent 75 to the foaming process. We subsequently computed the optical flow from one difference image to the next one in the sequence (algorithm available upon request to the authors). The optical flow algorithm applied was a multi-scale version of the Horn and Schunk algorithm. ${ }^{21}$ We chose this approach 80 for two reasons. Firstly, this method has a theoretical justification in terms of fluid dynamics. ${ }^{22,24}$ This is important, not only because we may be interested in tracing back the observed processes to extract physical conclusions, but because this ensures the consistency of the procedure in terms 85 of conservation of physical quantities. The second reason is that the method permits sub-pixel accuracy in the estimates, which is a must for the fast frame rates provided by synchrotron imaging. Simpler procedures such as correlationwindows approaches do not allow for sub-pixel estimates of 90 the velocity. 

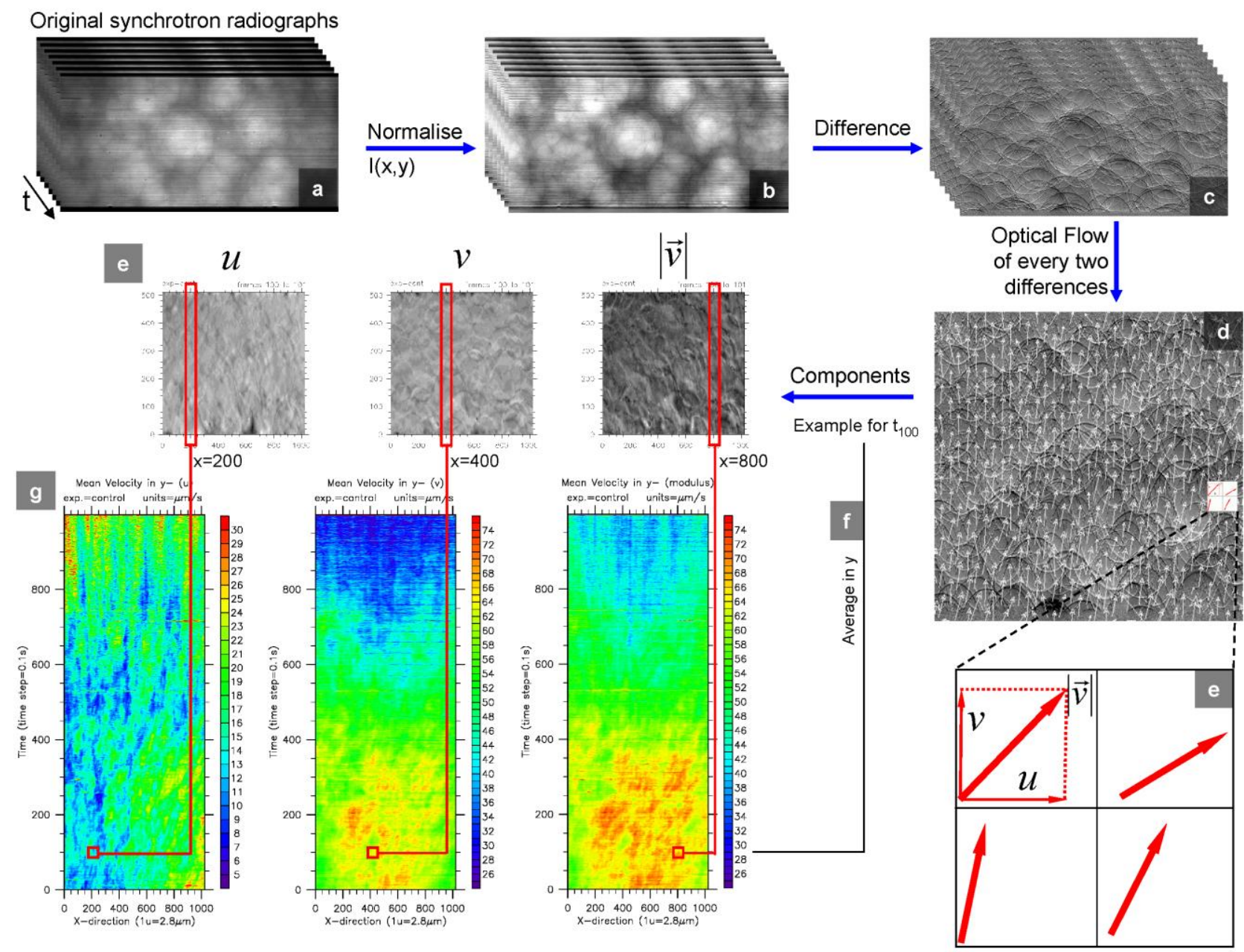

Fig. 1 Data analysis procedure. The successive steps and principles of the optical flow analysis are illustrated with the control sample. a, Original radiograph sequence. $\mathbf{b}$, Each radiograph is normalised to filter out both beam and optic artefacts. $\mathbf{c}$, Difference image between each radiograph and its successor, calculated to highlight changes. d, The optical flow from one difference image to the next in the sequence is calculated using a multi-scale 5 version of the Horn and Schunk algorithm (to facilitate visualisation, only 20 arrows per row have been plotted). e, The velocity vector obtained for each pixel is decomposed into its components: $\mathrm{u}, \mathrm{v}$ and the modulus, or magnitude, and $\mathbf{f}$, we calculated the average in the y-axis of the velocity $\mathbf{g}$, Diagram embedding the evolution of the velocities over time for each component.

The result of the optical flow algorithm was obtained in the 10 form of a vector field in which each pixel is attributed an associated velocity vector (Figure 1). The vector represents the most likely, physically-consistent motion of the foam at each point. The velocity vector can be decomposed into the $\mathrm{u}$ and $\mathrm{v}$ - component which respectively provide information on 15 the horizontal and vertical velocities of the flow. Due to the vertical expansion of the foam, the $v$ - component and the modulus, or magnitude, are very closely related. Given the small lateral sampling distances of the estimates, the field is sensitive to important topological changes such as bubble 20 coalescence and film break-up.

Our main contribution to the methodology is that to fully quantify these events we calculated the integral of the velocity in the preferred direction of foaming (y-axis) to obtain a mean velocity for the $\mathrm{x}$-direction. Repeating this process for each 25 difference image, we can build up a stacked diagram reflecting the temporal evolution of the velocities for each sample. This graph offers a fingerprint of the foaming process as it not only provides quantitative estimates of the foaming rate but also displays key topological transformations as point 30 and line discontinuities within the overall evolution. The latter is particularly important because it provides a new insight on the bubble rearrangement dynamics. ${ }^{5}$ The results obtained for three selected samples with different foaming behaviour are demonstrated in the following section. 


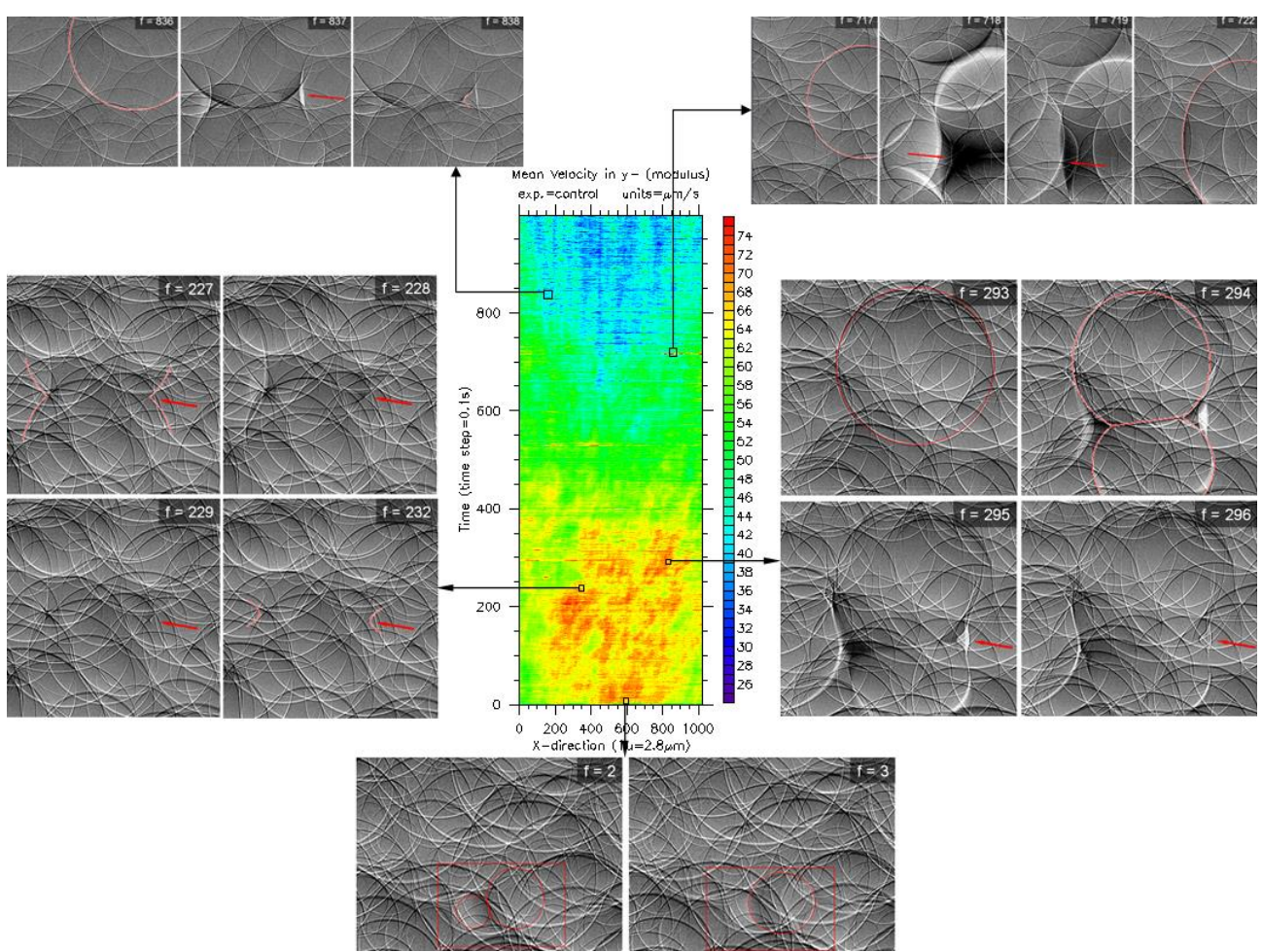

Fig. 2 The evolution of foaming of the control sample. The diagram represents a fingerprint of the foaming process, permitting the exact localisation of coarsening events and precise estimates of the foaming rate. A series of difference images has been included to show some of the events identified. frame 2-3, Ostwald ripening. frame 227-232, Coalescence due to capillary drainage. frame 293-296, Coalescence due to film rupture. frames 717-722 and 836$5 \mathbf{8 3 8}$, Coalescence due to film rupture. To clarify the topological changes, the initial and final bubbles have been outlined in red and the intermediate stages are marked with a red arrow.

\section{Results}

Figure 2 shows the diagram and corresponding images of some of these topological transformations for the control 10 (unfilled) sample. An examination of the images confirms bubble growth and motion and the existence of different physically-driven processes ranging from diffusion events from smaller to larger bubbles (Ostwald ripening) (Figure 2, frames 2-3) to the coalescence of adjoining bubbles due to 15 both capillary drainage (Figure 2, frame 227-232) and sudden film rupture (Figure 2, frame 293-296). As foaming progresses the system slows down revealing isolated events of interest, mainly film ruptures that result in coalescence (Figure 2, frame 717-722 and 836-838). After approximately ${ }_{20} 80 \mathrm{~s}$, the evolution has almost concluded. We can gain further information by studying the $u$ and $v$ components (Figure $3 a$ ). The $\mathrm{v}$ component reveals the upward motion resulting from the expansion of foam, which clearly stops after $80 \mathrm{~s}$. Meanwhile, the $\mathrm{u}$ component remains relatively constant 25 throughout the expansion stage. By the end of the stage, it shows cell rearrangements and film ruptures between adjacent cells. The presence of events in the $u$ but not in the $v$ diagram, and vice versa, suggests that the event has a determined directionality. Additionally, for this particular sample, there is 30 a clear rightward tendency for the u component, which could be due to a capillarity effect near the interface with the sample container. The same phenomenon is observed in the CNT sample, although in this case, the tendency is towards the left, where the meniscus of the liquid is still visible, indicating the
35 presence of the border. This directionality of foaming concludes towards the end of the experiments as the foams solidify.

The diagrams relating to the filled samples showed significant differences in the evolution of foaming (Figure $3 b$ 40 and c). Filled samples of both CNT and FGS evolved more slowly than the control sample. This slow evolution is verified by the initial images of the two systems, which show the presence of the liquid meniscus which has already disappeared from the field of view of the detector in the case of the control 45 sample (Figure 4). The growth patterns of the filled samples are quite similar. They both expand ( $\mathrm{v}$ component) at a low, and almost constant, rate and stop after about $50 \mathrm{~s}$ in the case of the CNT sample and around $60 \mathrm{~s}$ in that of the FGS sample. A plausible explanation for this slow and reduced evolution 50 could relate to an increase in the viscosity of the initial reactants. This increased viscosity would have two effects on the evolution of foaming. First, it would initially provide more resistance to the evolved gas, hence reducing the growth rate and expansion. Second, it would retard film thinning due 55 to drainage, hence increasing film stability. ${ }^{13}$ As in the control sample, by the end of the experiments, the $u$ component displays the events that occur, which are comparatively intense in the CNT sample. The large number of events in the CNT sample could represent a cooperative phenomenon, i.e. a 60 single bubble rupture that triggers an avalanche of ruptures. ${ }^{25}$ These ruptures could be due to the non-wetting surface of the filler, which has a destabilising effect on foams through a 
bridging-dewetting mechanism. ${ }^{26}$ Meanwhile, the hydrophilic nature of the FGS particles will have a tendency to locate at the bubble interface, thereby reducing film ruptures and coalescence. $^{27}$ The changes observed in the cellular structure 5 of the CNT and FGS samples could therefore be related to different phenomena. For the CNT sample, the dominant phenomena could be the film ruptures that occur at the end of the process. In contrast, the principal phenomena for the FGS sample could be diffusive coarsening due to slow growth.
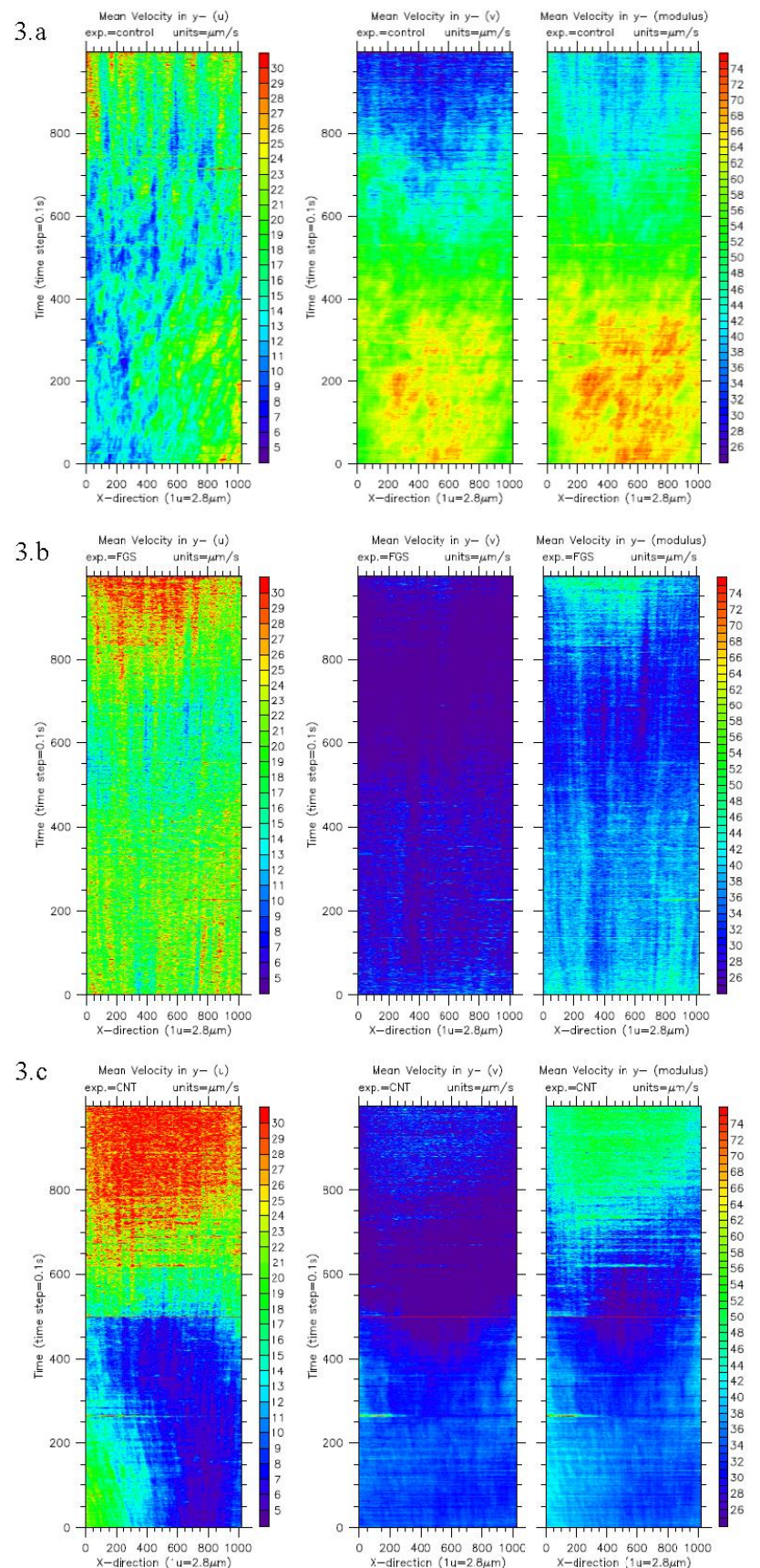

Fig. 3 Foaming diagrams for all samples. The diagrams show significant differences in the evolution of foaming for: a control, b FGS and c CNT samples. The red line observed in frame 500 of the CNT sample is due to a $5 \mathrm{~ms}$ delay in the experimental acquisition of the 15 radiographs. ["exp." stands for experiment and identifies the foam been analysed; i.e. control, FGS or CNT foam.]
A concise means of characterising the growth evolution would involve calculating the evolution over time of the mean modulus velocity for the whole frame. Figure 4 shows the 20 modulus velocity of the three samples and provides a general view of the process stated above: i) the slower evolution of filled samples, ii) the growth patterns or steps and iii) the occurrence of key topological changes.
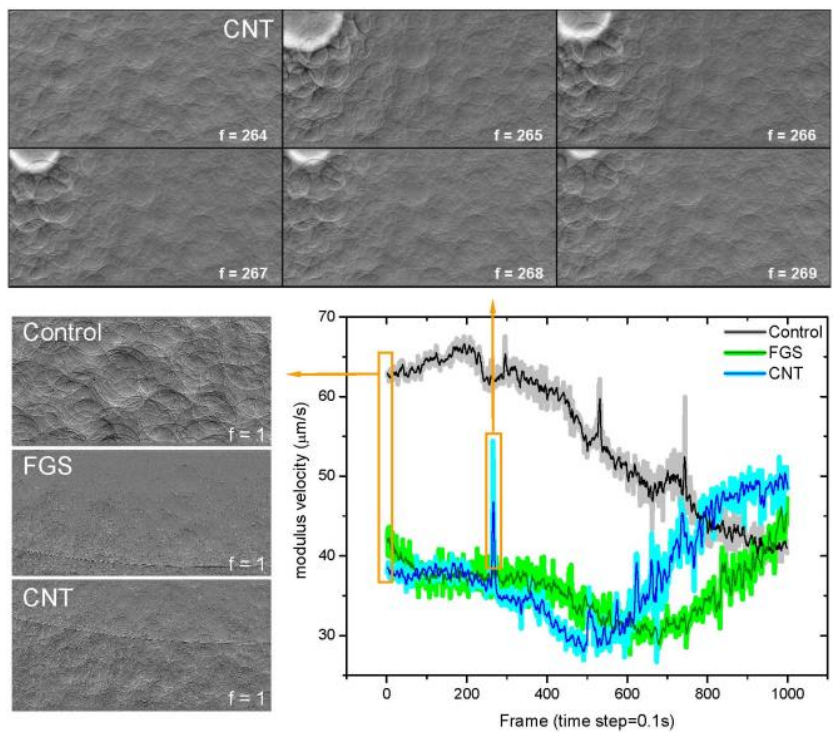

${ }_{25}$ Fig. 4 Time evolution of the mean modulus velocity for all samples. This curve is the most concise way to represent the characteristics of the different processes, as it provides their respective foaming rates and a general overview. The thick shaded lines show the calculated mean modulus velocities and the thin dark lines show the average value for 5

30 adjacent data points. The difference images show the initial states of all three samples and the film rupture in the CNT sample.

\section{Conclusions}

The method presented here was based on linear expansion of the Navier-Stokes equations and was used to the detailed 35 analysis of the observed image sequences. The algorithm can be universally applied to characterise the dynamics of multiphase systems by means of a "fingerprint" diagram showing changes in the optical flow field. These diagrams allow a straightforward identification of events, their duration, 40 and the time between them, as well as both general and local flow patterns and velocities.

In this paper, we presented experimental results about the evolution of reactive polymer foams, in the process of their formation, expansion and solidification. Synchrotron 45 radiography was needed to observe the evolution of the foam with very high resolution, and to study the effects of two different types of solid fillers in the foaming medium hydrophobic carbon nanotubes (CNT) and functionalized graphene sheets (FGS).

${ }_{50}$ We found that the nanofillers significantly affected the dynamics of foaming. The filled samples evolved more slowly than the control sample as the result of an increase in the viscosity of the initial reactants. Moreover, the foaming evolution of the filled samples showed differences in the 55 expansion and solidification processes (cfr. the colour differences in the three parts of figure 3). While the CNT 
sample exhibited a large number of topological transformations at the end of the foaming process, the FGS sample was noticeably less eventful. Such different behaviour would be related to the surface nature of the fillers. The 5 physical explanation is that non-wetting surface of the CNT had a destabilising effect on foams through a bridgingdewetting mechanism resulting on film bubble ruptures. In contrast, the hydrophilic nature of the FGS particles had a stabilising effect on foams due to their tendency to locate at 10 the bubble interface, thereby reducing film ruptures and coalescence. Hence, the main coarsening phenomena in the CNT sample would be film ruptures at the end of the foaming process. Regarding the FGS sample, the principal phenomena should be diffusive coarsening due to slow growth.

15 This theoretical behaviour is consistent with the empirical results in the fingerprint diagrams. The methodology presented here provides a mean to improve the understanding of the dynamics of reactive foaming and how fillers affect the foaming evolution. Further study of the data presented in this 20 paper will be carried out in order to analyse the spatiotemporal rearrangements of the bubbles. In addition, it would be interesting to distinguish among the different topological transformations from the fingerprint diagram by analysing different types of foams or multiphase systems.

25 As a closing remark, the method is not limited to synchrotron imaging or foaming processes. Rather, it could be applied to a variety of instruments and materials such as video sequences of the evolution of multiphase systems, providing robust characterizations of the process under investigation.

\section{${ }_{30}$ Acknowledgements}

We thank the European Synchrotron Radiation Facility (ESRF, beamline ID19) for providing beam time and support (MA641). RV acknowledges a JAEDoc contract from CSIC. The work at ICTP-CSIC was supported by the Spanish ${ }_{35}$ Ministry of Science and Innovation under project MAT 2007 61116. The work at the UCLM was funded by research grants PAI06-0102-7466 (Regional Government of Castilla-La Mancha), CGL2006-03611 (MiCInn), and the Ramon y Cajal programme.

\section{${ }_{40}$ Notes and references}

a Institute of Polymer Science and Technology, (CSIC), Juan de la Cierva, 3, 28006 Madrid, Spain, Fax: 3491564 4853; Tel: +34 91258 7424; Email:rverdejo@ictp.csic.es.

${ }^{b}$ Institute of Environmental Sciences (ICAM), Department of

45 Environmental Sciences, University of Castilla-La Mancha (UCLM),

Toledo, Spain.E-mail: francisco.tapiador@uclm.es.

${ }^{b}$ ANKA/Institute for Synchrotron Radiation, Forschungszentrum Karlsruhe, Germany.

$\dagger$ Electronic Supplementary Information (ESI) available: Movies: S1 50 Foaming recorded by standard video camera. S2 Foaming recorded by high resolution radiography. See DOI: 10.1039/b000000x/

1. R. K. Prud'homme and S. A. Khan, eds., Foams: Theory, Measurements and Applications, CRC Press, New York, 1996.

55 2. L. J. Gibson and M. F. Ashby, Cellular Solids: Structure and Properties 2nd edn., Cambridge University Press, 1997.
3. S. A. Koehler, S. Hilgenfeldt and H. A. Stone, Langmuir, 2000, 16, 6327-6341.

4. D. J. Durian, D. A. Weitz and D. J. Pine, Science, 1991, 252, 686$60 \quad 688$.

5. A. S. Gittings and D. J. Durian, Phys. Rev. E, 2008, 78, 066313

6. S. Cohen-Addad and R. Höhler, Phys. Rev. Lett., 2001, 86, 47004703.

7. D. Weaire, S. Findlay and G. Verbist, J. Phys.: Condens. Matter, 65 1995, 7 L217-L222.

8. D. L. Schmidt, in Foams: Theory, Measurements and Applications, eds. R. K. Prud'homme and S. A. Khan, CRC Press, New York, 1996, pp. 287-314.

9. J. Banhart, H. Stanzick, L. Helfen and T. Baumbach, Appl. Phys. 70 Lett., 2001, 78, 1152-1154.

10. S. Hilgenfeldt, S. A. Koehler and H. A. Stone, Phys. Rev. Lett., 2001, 86, 4704-4707.

11. D. Weaire and S. Hutzler, The Physics of Foams, Oxford University Press, Dublin, 1999.

75 12. D. Klempner and K. C. Frisch, Handbook of polymeric foams and foam technology, Hanser Publishers, 1991.

13. J. H. Saunders and R. H. Hansen, in Plastics Foams, eds. K. C. Frisch and J. H. Saunders, Marcel Dekker, New York, 1972.

14. R. Verdejo, F. Barroso-Bujans, M. A. Rodriguez-Perez, J. A. de Saja,

80 M. Arroyo and M. A. Lopez-Manchado, J. Mater. Chem., 2008, 18, 3933-3939.

15. R. Verdejo, F. Barroso-Bujans, M. A. Rodriguez-Perez, J. A. de Saja and M. A. López-Manchado, J. Mater. Chem., 2008, 18, 2221-2226.

85 16. A. G. MacPhee, M. W. Tate, C. F. Powell, Y. Yue, M. J. Renzi, A. Ercan, S. Narayanan, E. Fontes, J. Walther, J. Schaller, S. M. Gruner and J. Wang, Science, 2002, 295, 1261-1263.

17. J. R. Royer, E. I. Corwin, A. Flior, M. L. Cordero, M. L. Rivers, P. J. Eng and H. M. Jaeger, Nature Physics, 2005, 1, 164-167.

90 18. F. García-Moreno, A. Rack, L. Helfen, T. Baumbach, S. Zabler, N. Babcsán, J. Banhart, T. Martin, C. Ponchut and M. DiMichiel, Appl. Phys. Lett., 2008, 92, 134104

19. A. Myagotin, L. Helfen and T. Baumbach, Meas. Sci. Technol., 2009, 20, 055703

95 20. H. C. Schniepp, J. L. Li, M. J. McAllister, H. Sai, M. HerreraAlonso, D. H. Adamson, R. K. Prud'homme, R. Car, D. A. Saville and I. A. Aksay, J. Phys. Chem. B, 2006, 110, 85358539.

21. B. K. P. Horn and B. G. Rhunck, Artificial Intelligence, 1981, 17, 100 185-203.

22. F. J. Tapiador, Int. J. Remote Sens., 2008, 29, 5851-5862.

23. J. Banhart, ed., Advanced Tomographic Methods in Materials Research and Engineering, Oxford University Press, 2008.

24. D. Heitz, P. Héas, E. Mémin and J. Carlier, Exp. Fluids, 2008, 45, 105 595-608.

25. W. Müller and J.-M. di Meglio, J. Phys.: Condens. Matter, 1999, 11, L209-L215.

26. B. P. Binks, Curr. Opin. Colloid Interface Sci., 2002, 7, 21-41.

27. T. N. Hunter, R. J. Pugh, G. V. Franks and G. J. Jameson, $A d v$. 110 Colloid Interface Sci., 2008, 137, 57-81. 